\title{
Research on new photovoltaic grid connection device based on Freescale Carle MK60FN1M0 processor and Multi-mode wireless communication technology
}

\author{
Ge Leyi ${ }^{1, a}$, Deng Zuqiang ${ }^{1, b}$, Zhuang Jian ${ }^{2}$ \\ ${ }^{1}$ State Grid Corporation of China, Electric Power Research Institute. Beijing, 102200 \\ ${ }^{2}$ Tianjin Electric Power Company, State Grid, Tianjin, 300010 \\ ageleyi@sgepri.sgcc.com.cn, bdengzuqiang@sgepri.sgcc.com.cn
}

\begin{abstract}
Keywords: MK60FN1M0; Modbus; Photovoltaic inverter; Multi-mode wireless terminal; Grid connection device
\end{abstract}

\begin{abstract}
Design a new photovoltaic grid connection device based on Freescale MK60FN1M0 processor and Multi-mode wireless communication technology. In terms of Modbus protocol, MK60FN1M0 communicates with photovoltaic inverter, the device acquires measurement data such as: $\mathrm{DC}$ voltage, $\mathrm{DC}$ current, $\mathrm{AC}$ voltage, $\mathrm{AC}$ current, $\mathrm{AC}$ frequency, power of the photovoltaic inverter, and opening-closing state of air switch. These measurement data are stored in real-time in the processor flash. At the same time, MK60FN1M0 is connected with power Multi-mode wireless terminal by UART, to guarantee the acquired measurement data transferred to remote power automation master station according to pre-set Modbus protocol in high speed and high efficiency.
\end{abstract}

\section{Introduction}

Because of the energy crisis and environmental pollution problems have become increasingly prominent, new energy power is needed to develop. The sunlight as a public, environment-friendly, renewable resource has its unparalleled advantage, it more and more received developer's increasing attention.

At present, photovoltaic grid connection devices on the market have many technical problems, for example, its information level in the relatively backward status, low voltage lines do not have the communication channels generally, it's metering and billing cannot realize in two directions etc. The current market needs a new photovoltaic grid connection device, which not only can reduce the construction difficulty of new energy photovoltaic power generation project, reduce the overall cost of the project, but also ensure the safe and stable operation of regional new energy power grid.

For improvement of photovoltaic grid connection device, scholars made a lot of constructive work inside and outside China. For example: Guo Yongji and Lv Guofang studied optimization problems and debugging problems of communication system based on Modbus protocol [1, 2]. Zhou Jinghua studied low voltage ride-through control problems [3]. Liu Dongran made a review on models for photovoltaic generation system [4]. Zhao Qinglin solves several problems on inverter control technology [5]. Donghui described some key problems of photovoltaic power generation system [6]. At the same time, U. Herrmann, Benjamin Sahan and Yoshihiro Konishi, discusses some technical problems of photovoltaic inverter design and application [7-9], N. Pogaku studied micro grid modeling, analysis and test which based on the photovoltaic inverters [10]. These China domestic and foreign researches put forward our technology improvement of photovoltaic grid connection device.

This paper designed a new photovoltaic grid connection device based on Freescale Carle MK60FN1M0 processor and Multi-mode wireless communication technology. In the second chapter, this paper introduces the technical principle of the device. In the third chapter, this paper introduces the technical realization details of the device from four aspects: main components of the device, communication protocol, Data transmission process and electrical cable wirings. In the last chapter, a summary of the device innovation points is carried out. 


\section{Technical principle}

Technical principle of new photovoltaic grid connection device is described in Fig.1.

Direct current (DC), generated by the photovoltaic battery, is connected to the new photovoltaic grid connection device. The device consists of air switch, inverter, air switch, lightning arrester, MK60FN1M0 circuit board and Multi-mode wireless terminal. Alternating current (AC), generated by the inverter, is measured by Electric energy meter, and connected to $220 \mathrm{~V}$ power grid. At the same time, MK60FN1M0 circuit board is powered by 5V DC, which is generated by Ld-Harvest switching power supply. In terms of Modbus protocol, MK60FN1M0 circuit board communicates with the inverter use 232 serial port. MK60FN1M0 circuit board acquires all kinds of measurement data collected by sensors, and transfer these measurement data to remote power automation master station by $3 \mathrm{G} / \mathrm{Wi}-\mathrm{Fi} / \mathrm{Mesh} /$ Wired Ethernet .

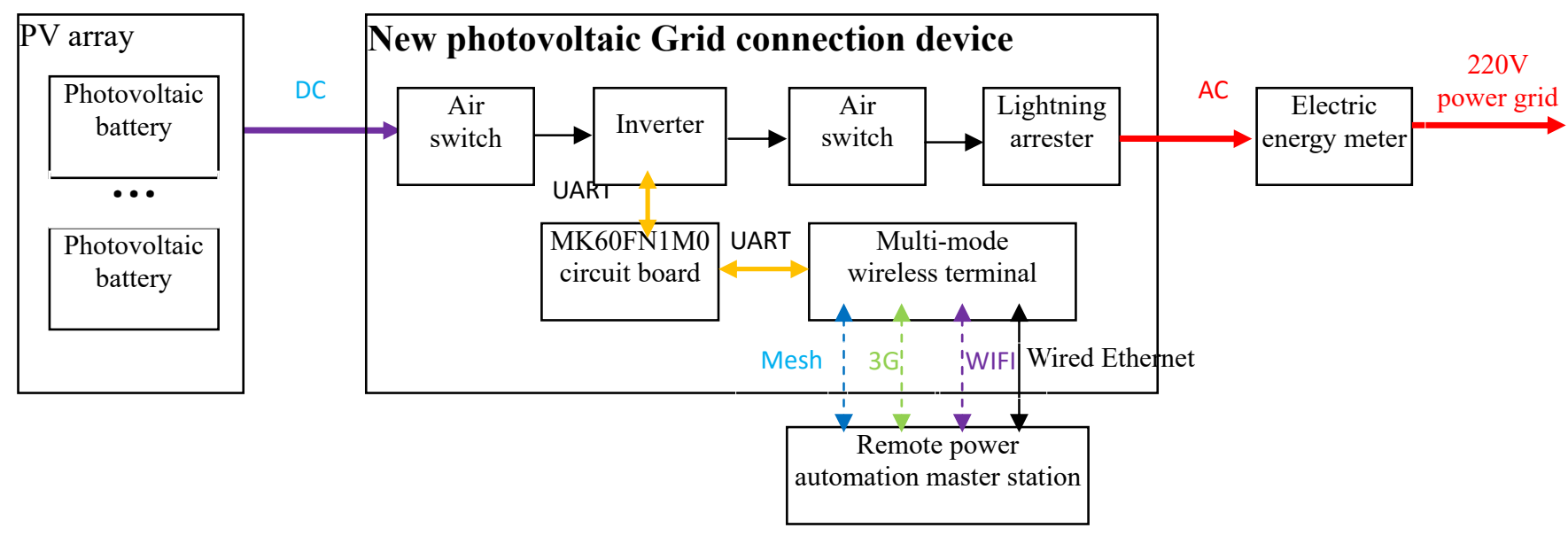

Fig.1 Technical principle of new photovoltaic grid connection device

\section{Technical realization}

Main components

(1) Inverter

The function of inverter is converting the DC power into AC power, it is a key component of the new photovoltaic grid connection device, and it contains several modules, such as: signal detection module of power system, output current control module, maximum power point tracking module (MPPT), anti-islanding module and other protection modules. Some of its key technical parameters are as follows:

Maximum input power: $5400 \mathrm{~W}$.

Input voltage range: $150 \mathrm{~V}-550 \mathrm{~V}$.

Maximum input current: $26 \mathrm{~A}$.

Rated output power: $4600 \mathrm{~W}$.

Maximum output power: $5100 \mathrm{~W}$.

Maximum output current: $22 \mathrm{~A}$.

Rated power grid voltage: 230 Vac.

Power grid voltage range: 180 Vac- 276 Vac.

Rated power grid frequency: $50 \mathrm{~Hz}$.

Maximum efficiency: $97.60 \%$.

Protection level: IP65.

Operating temperature range: $-25 \sim 60^{\circ} \mathrm{C}$.

(2) Design of MK60FN1M0 circuit board

MK60FN1M0 circuit board includes several parts: switching power supply circuit, $5 \mathrm{~V} / 3.3 \mathrm{~V}$ circuit, GPIO input circuit, FC-RF209 (Mesh) circuit, ESP8266 circuit, MK60FN1M0 core board interface circuit, etc. 
1)Switching power supply circuit

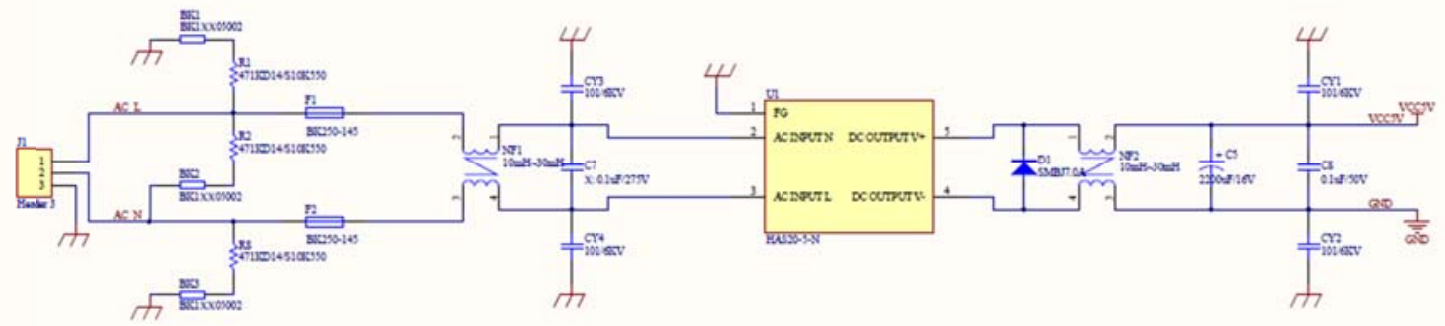

Fig.2 Switching power supply circuit

The function of switching power supply circuit is converting $220 \mathrm{~V}$ AC power into $5 \mathrm{~V}$ DC power by Ld-Harvest switching power supply module from $220 \mathrm{~V}$ AC power grid. Switching power supply circuit is described in Fig.2.

2) $5 \mathrm{~V} / 3.3 \mathrm{~V}$ circuit

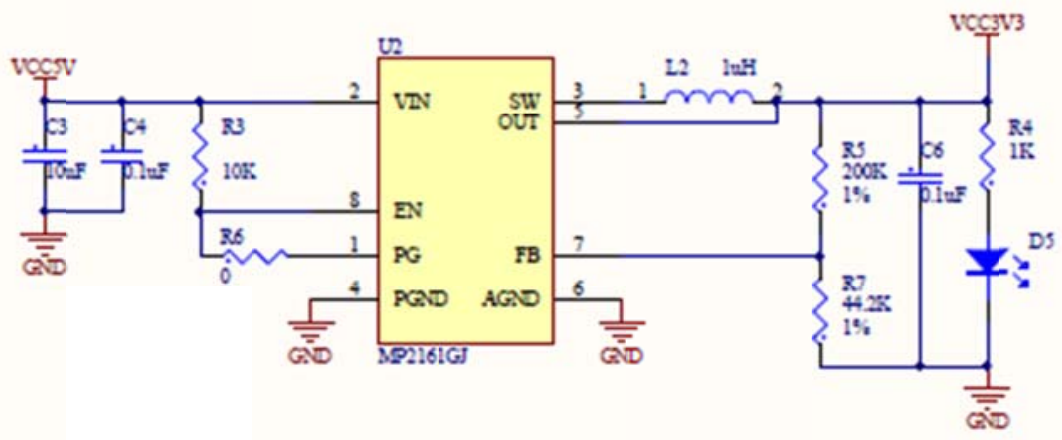

Fig. 3 VV/3.3V circuit

The function of $5 \mathrm{~V} / 3.3 \mathrm{~V}$ circuit is converting $5 \mathrm{~V}$ DC into $3.3 \mathrm{~V} \mathrm{DC}$, which supplies power for the FC-RF209 module and the ESP8266 module. 5V/3.3V circuit is described in Fig.3.

3) GPIO input circuit

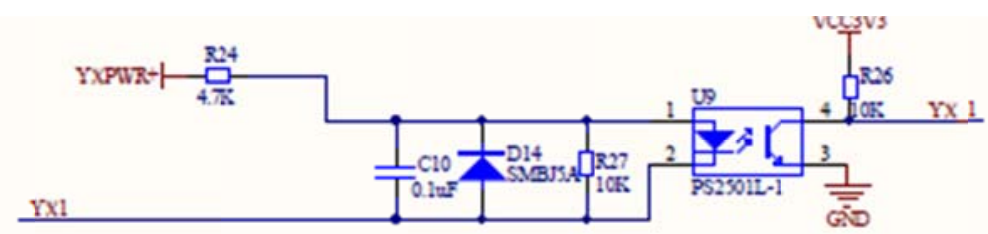

Fig.4 GPIO input circuit

The function of GPIO input circuit is acquiring remote signal, all the signals Opt coupler isolation [11]. GPIO input circuit is described in Fig.4.

4) FC-RF209 (Mesh) circuit

The function of FC-RF209 (Mesh) circuit is making the FC-RF209 module communicates normally, which can send and receive MK60FN1M0 core board serial data. FC-RF209 (Mesh) circuit is described in Fig.5.

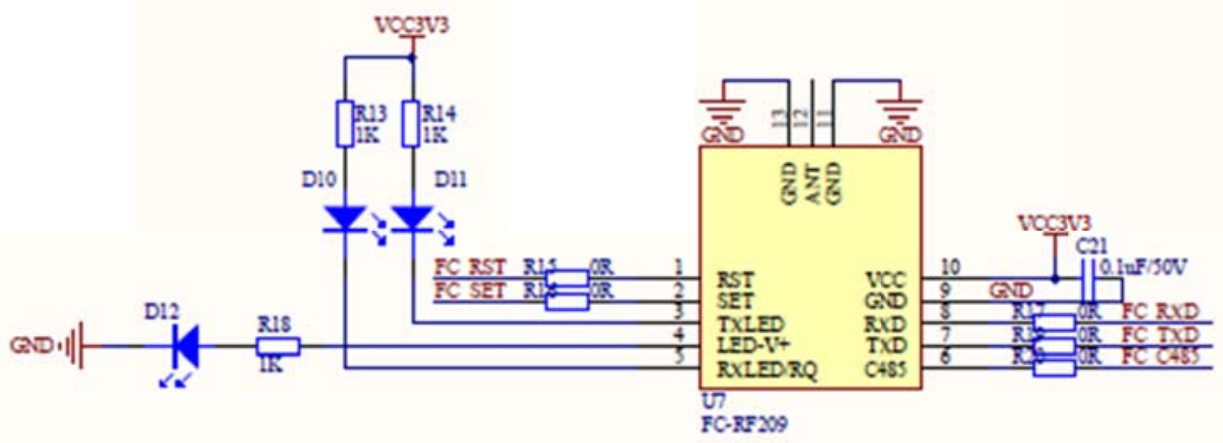

Fig.5 FC-RF209 (Mesh) circuit 
5)ESP8266(Wi-Fi)circuit

The function of ESP8266(Wi-Fi)circuit is making the ESP8266 module communicates normally, which can send and receive MK60FN1M0 core board serial data. ESP8266(Wi-Fi)circuit is described in Fig.6.

ESP8266(Wi-Fi)circuit is described in Fig.6.

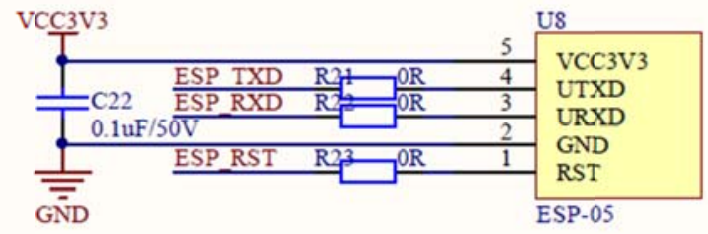

Fig.6 ESP8266(Wi-Fi)circuit

6) MK60FN1M0 core board interface circuit

The function of MK60FN1M0 core board interface circuit is making five UART pins, two GPIO pins, VCC, GND and other pins of the MK60FN1M0 core board connect with the other circuit $[12,13]$. MK60FN1M0 core board interface circuits are described in Fig.7 (A), (B).

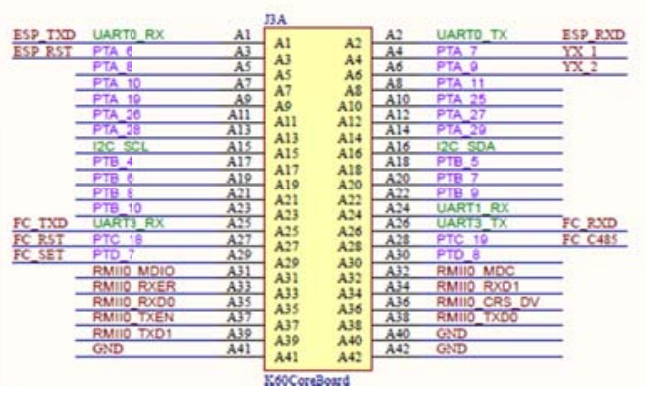

(A)

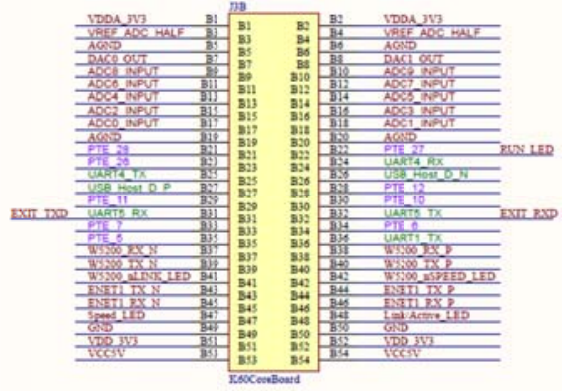

(B)

(3) Multi-mode wireless terminal

Fig.7 MK60FN1M0 core board interface circuit

Multi-mode wireless terminal includes communication strategy selection module, wireless Mesh module (LTE230 wireless communication), Wi-Fi module, GPRS module, 3G module and other modules. Its uplink and downlink communication modes are shown in Figure 8. The uplink communication support four communication modes: GPRS, 3G, Mesh, Wi-Fi, it connects with public base station by Mesh and Wi-Fi, and connects with special base station by GPRS and 3G. The public base station and the special base station communicate with remote electric power automation master station by Ethernet to realize the function of remote control, remote measuring and remote communication. Its downlink communication realizes data interaction with MK60FN1M0 circuit board by the UART.

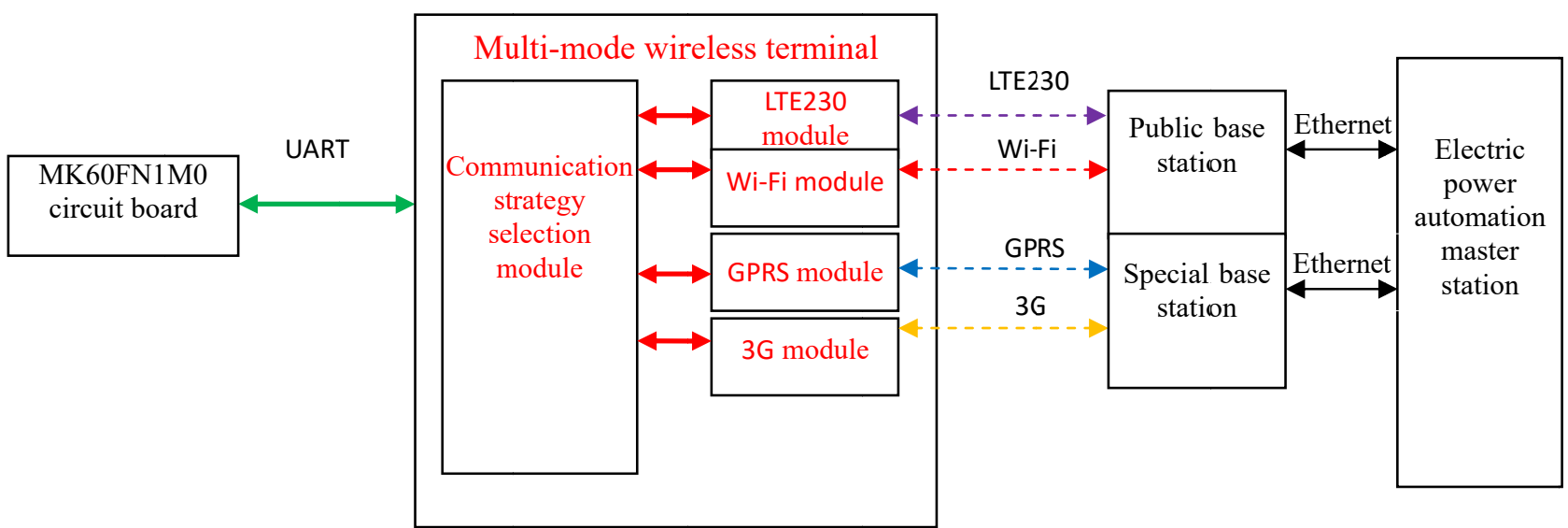

Fig.8 uplink and downlink communication modes of Multi-mode wireless terminal

\section{Communication protocol}

Up and down communication protocol message format are described in Fig. 9 below: 


\begin{tabular}{|c|c|c|c|c|c|c|c|}
\hline Header & $\begin{array}{c}\text { Source } \\
\text { address }\end{array}$ & $\begin{array}{c}\text { Destination } \\
\text { address }\end{array}$ & $\begin{array}{c}\text { Control } \\
\text { code }\end{array}$ & $\begin{array}{c}\text { Function } \\
\text { code }\end{array}$ & $\begin{array}{c}\text { Data } \\
\text { length }\end{array}$ & Data0,1,2.. & Checksum \\
\hline
\end{tabular}

In Fig. 9:

Fig. $9 \mathrm{Up}$ and down communication protocol message format

Header (0x55 0xAA): the header of each packet.

Source Address: designate the sender address. (it is XX00 for MK60FN1M0, or it is 00XX for Inverter).

Destination address: designate the receiver address.(it is XX00 for MK60FN1M0, or it is 00XX for Inverter).

There are 4 kinds of control code: 1) Register (0x00); 2) Read (0x01); 3) Write (0x02); 4) Execute (0x03).

Function code: Detail function description.

Data length: designate the data length. (If there is not the data column, the data length is 0 ).

Data0,1,2..: Data column.

Checksum: Header+Source Address+Destination Address+Control Code+Function Code+Data length + Data $0+\ldots+$ Data $(\mathrm{N}-1)$.

\section{Data transmission process in $\mathrm{C}$ language}

Data transmission of the new photovoltaic grid connection device in use of $\mathrm{C}$ codes can be described in following: at first, initialize the UART 0-6, flash and RTC, send messages of registration request and confirmation, judge whether or not the registration is correct, and then send commands to read inverter dada of remote control, remote measuring and remote communication. Secondly, determine parameter index and value of data, and make the important parameters' values to be stored to the flash. Finally, send registration packet and the confirmation message of UART $1-5$, and judge whether or not the registration is correct, then transfer the parameters' values of the real-time data and the historical data stored in flash according to the demand from electric power automation master station. Data transmission process realized by $\mathrm{C}$ codes can be shown in Fig. 10 .

\section{Electrical cable wirings}

The important electrical cable wirings of the new photovoltaic grid connection device include two parts: the AC side cable wiring connection and the inverter cable wiring connection:

1)AC side cable wiring connection

For Ld-Harvest switching power supply module, one cable is connected to $220 \mathrm{~V} \mathrm{AC}$ grid, the other cable is connected to MK60FN1M0 circuit board. For air switch in the AC side, one cable is connected to $220 \mathrm{~V}$ AC grid, another cable is connected to photovoltaic inverter, and the other cable is connected to lightning arrester. For lightning arrester, one cable is connected to the air switch in the AC side; the other cable is connected to ground.

\section{To $220 \mathrm{~V}$ AC grid}

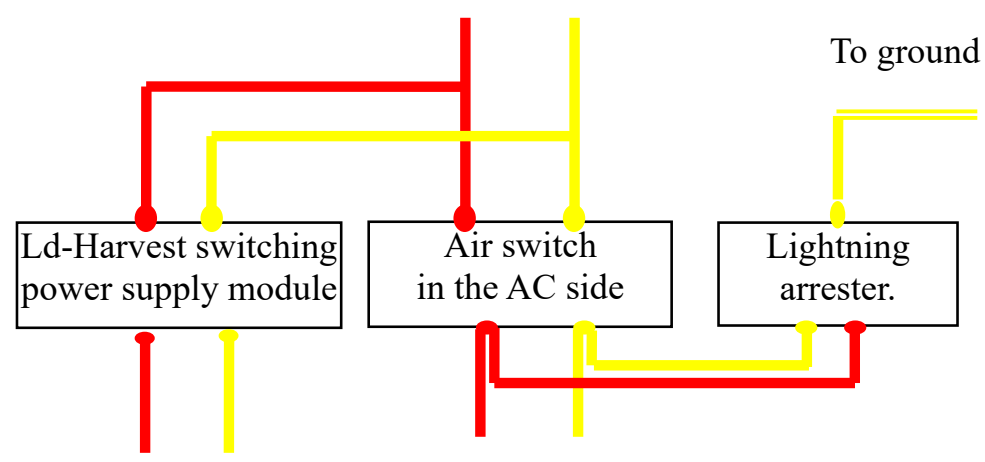

To MK60FN1M0 circuit board

To photovoltaic inverter

2)Photovoltaic inverter cable wiring connection

For the photovoltaic inverter, one cable is connected to air switch in the AC side; the other cable is connected to air switch in the DC side. The photovoltaic inverter is connected with MK60FN1M0 
circuit board by UART.

Air switch in the $\mathrm{AC}$ side
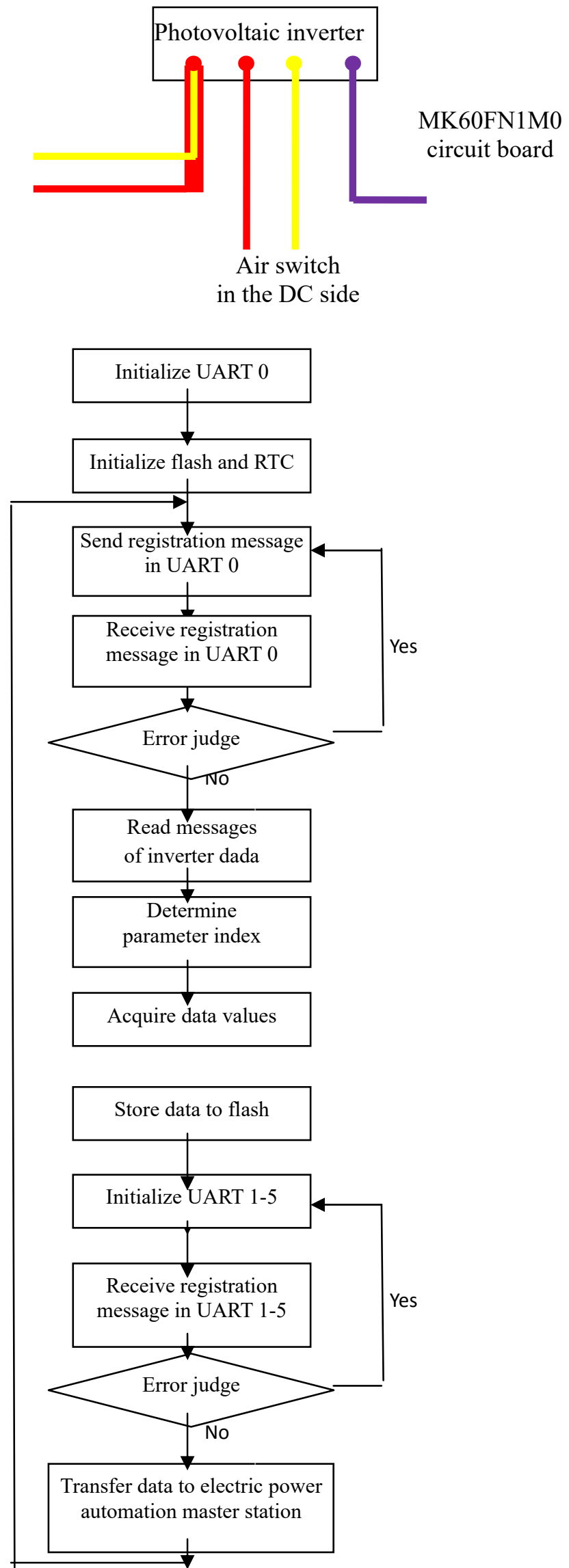

Fig. 10 Data transmission process realized by $\mathrm{C}$ codes 


\section{Summary}

This paper designed a new photovoltaic grid connection device based on Freescale Carle MK60FN1M0 processor and Multi-mode wireless communication technology. Innovation points of the new device lies in following: 1.Use Freescale MK60FN1M0 processor, power Multi-mode wireless terminal, advanced metering infrastructure, Modbus protocol, collect the running status information of the new device.2. Use power Multi-mode wireless terminal to guarantee measurement data transferred to remote power automation master station by $3 \mathrm{G} / \mathrm{Wi}-\mathrm{Fi} / \mathrm{Mesh} / \mathrm{Wired}$ Ethernet in high speed and high efficiency. After mathematical modeling and analysis, monitor all photovoltaic grid connection devices running in the region. The new photovoltaic grid connection device not only realizes a convenient connection of photovoltaic power to grid, but also realizes preventive maintenance of the photovoltaic grid connection device.

\section{References}

[1] GUO Yongji, WANG Xinggui. Real ization of the Communication between IPC and Intelligent Instrument Based on Modbus Protocol.Journal of Gansu Sciences,2008, 20(1):102-104(in Chinese).

[2] LU Guofang, TANG Hailong, LI Jin. Implementation of Serial Port Debugging Software Based on Modbus RTU Protocol. Computer technology and development.2009,19(9):236,237, 241(in Chinese).

[3] ZHOU Jinghua, LIU Jindong, CHEN Yaai, LI Jin. Low Voltage Ride-Through Control of High Power Inverter for Gird-Connection of Photovoltaic Generation. Power System Technology. 2013, 37(7):1879-1807 (in Chinese).

[4] Liu Dongran, Chen Shuyong, Ma Min, et al. A review on models for photovoltaic generation system[J]. Power System Technology, 2011, 35(8): 47-52(in Chinese).

[5] Zhao Qinglin, Guo Xiaoqiang, Wu Weiyang. Research on control strategy for single-phase grid-connected inverter[J]. Proceedings of the CSEE, 2007, 27(16):60-64(in Chinese).

[6] LI Donghui, WANG Hexiong, ZHU Xiaodan, etal. Research on several critical problems of photovoltaic grid-connected generation system[J]. Power System Protection and Control, 2010, 38(21): 208-214(in Chinese).

[7] Herrmann U, Langer G, Broeck H. Low cost DC to AC converter for photovoltaic power conversion in residential applications[C] // Proc IEEE PESC, 1993: 588-594.

[8] Benjamin Sahan, Antonio Notholt Vergara, Norbert Henze, et al. A single-stage PV module integrated converter based on a low-power current-source inverter[J]. IEEE Transactions on Industrial Electronics, 2008, 55(7): 2602-2608.

[9] Yoshihiro Konishi, Yung fuHuang, Min Juhsieh. Utility-interactive high-frequency flyback transformer link three-phase inverter for photovoltaic AC module[C] // Industrial Electronics 35th Annual Conference of IEEE, 2009: 937-942.

[10] N. Pogaku, M. Prodanovic, T. C. Green. Modeling, Analysis and Testing of Autonomous Operation of an Inverter-Based Microgrid. IEEE Trans on Power Electronics,2007, 22(2):613-625.

[11] Khan Z.A.,Volakis J.L.. Experimental and Analytical Study of EMC/EMI Effects on PCBs and Cables Enclosed within Metallic Structures[C]. Antennas and Propagation Society International Symposium, 2006:49-52..

[12] ARM. Cortex-M4 Technical Reference Manual.pdf[DB/OL]. http://www.arm .com.

[13] Freescale Semiconductor Inc. K60 Data Sheet.pdf[DB/OL]. http://www.freescale.com. 